\title{
非共价作用在过渡金属催化的选择性碳氢键活化中的应用
}

\author{
廖港吴勇杰史炳锋* \\ (浙江大学化学系 杭州 310027)
}

\begin{abstract}
摘要 过渡金属催化的碳氢键活化是合成有机化合物最有效的工具之一，基于底物本身官能团或者共价键连接的导向 基策略是目前实现碳氢键选择性活化的主要手段. 非共价作用在分子生物学、超分子化学、材料科学及药物研发中具 有重要意义, 近年来, 非共价作用也被应用于过渡金属催化的惰性碳氢键的选择性活化. 本文总结了非共价作用在选 择性碳氢键活化领域的研究进展，并按照非共价键的作用类型，将其分为氢键作用、离子对作用、路易斯酸碱对作用 和静电作用等，探讨了催化体系中心金属、配体和底物间相互作用力的模式，并展望了未来研究工作的方向.
\end{abstract}

关键词 非共价键作用; 选择性; 碳氢键活化; 瞬态导向

\section{Noncovalent Interaction in Transition Metal-Catalyzed Selective C-H Activation}

\author{
Liao, Gang Wu, Yong-Jie Shi, Bing-Feng* \\ (Department of Chemistry, Zhejiang University, Hangzhou 310027)
}

\begin{abstract}
Transition metal-catalyzed direct $\mathrm{C}-\mathrm{H}$ functionalization is one of the most efficient and powerful tools for the rapid synthesis of organic molecules. The use of functional groups in the molecules or covalently attached coordinating groups as directing groups has been realized as a major strategy to control the selectivity. Noncovalent interactions are of great importance in the field of molecular biology, supramolecular chemistry, material science and drug discovery. More recently, the use of well-designed ligands to enable the site-selective $\mathrm{C}-\mathrm{H}$ functionalization via noncovalent interactions has emerged as a highly promising yet relatively less explored strategy. In this perspective, recent advances in this cutting-edge area are summarized. The perspective was classified into four sections according to the type of noncovalent interactions, including hydrogen bonding, ion pair, Lewis acid-base interaction and electrostatic interaction. Emphasis is placed on the mode of noncovalent interactions among the transition metals, ligands and substrates. The limitation of current research and the prospect of future work will also be discussed. We anticipate that this strategy might become a promising complementary strategy to control the positional selectivity in $\mathrm{C}-\mathrm{H}$ functionalization reactions.

Keywords noncovalent interaction; selectivity; $\mathrm{C}-\mathrm{H}$ activation; transient directing group
\end{abstract}

\section{1 引言}

近年来, 过渡金属催化的碳氢键官能团化反应成为 有机合成领域的研究热点之一 ${ }^{[1]}$. 相对于传统偶联反应, 其无需对起始原料进行预官能团化, 能够将惰性碳氢键 切断, 进行直接官能团化, 从而具有原子经济性和步骤 经济性等优点, 受到化学家们的广泛关注. 然而, 有机 分子中常常具有多个化学环境相似的碳氢键, 要实现特 定碳氢键的官能团化是极具挑战性的. 为了对碳氢键活 化位点进行精准控制, 目前最常用的策略是利用底物本 身配位官能团或者共价键连接的配位基团, 即导向基策 略. 导向基团与过渡金属进行配位后, 通常通过动力学 有利的五元环金属中间体, 选择性活化邻近位置的碳氢
键, 实现特定位点选择性(Scheme 1a) ${ }^{[2]}$. 然而, 引入的 导向基团通常需要额外的步骤进行连接和脱除, 这就使 得合成步骤变长, 成本增加, 也大大降低了反应的效率. 为了更好地解决这一问题, Yu 课题组开创性地发展了基 于可逆共价键的 “瞬态导向策略” ${ }^{[3]}$, 在实现位点选择 性精准控制的同时，避免了传统共价键连接的导向基团 的连接和脱除(Scheme $1 \mathrm{~b})^{[4]}$. 除了以上策略, 非共价键 策略近年来也初现端倪, 为惰性碳氢键官能团化提供了 一种全新的思路 ${ }^{[5]}$. 在本文中, 我们将从非共价键在选 择性碳氢键官能团化的应用角度进行阐述, 希望对该领 域的研究提供参考和展望.

一般而言，在导向碳氢键活化过程中，强的导向基 团和过渡金属作用使得反应具有更高的选择性，然而，

*E-mail: bfshi@zju.edu.cn; Tel.: 0086-571-88981229

Received February 8, 2020; published March 12, 2020.

Project supported by the National Natural Science Foundation of China (Nos. 21901228, 21772170), the China Postdoctoral Science Foundation (No. 2019M650135), the Outstanding Young Talents of Zhejiang Province High-level Personnel of Special Support (No. ZJWR0108) and the Natural Science Foundation of Zhejiang Province (No. LR17B020001).

项目受国家自然科学基金(Nos. 21901228, 21772170)、中国博士后科学基金(No. 2019M650135)、浙江省万人计划青年拔尖(No. ZJWR0108)和浙江省 自然科学基金(No. LR17B020001)资助. 
由于形成的环金属中间体具有相对更强的稳定性，因此 在一定程度上, 这种强作用力也可能对反应活性有一定 的影响. 作为互补, 非共价键在选择性碳氢健活化中的 作用日益凸显出来. 非共价键一般包括氢键、静电作用、 离子对作用、 $\pi-\pi$ 堆积效应、范德华力和疏水作用力等. 非共价键作用常见于生物系统和有机合成之中, 例如, 非共价键在有机小分子催化、酶催化、超分子化学和药 物开发中都起着重要的作用 ${ }^{[6,7]}$. 在这里主要介绍过渡 金属与非共价键共同作用的选择性碳氢键官能团化反 应. 在该策略中, 配体的官能团与底物分子的官能团通 过非共价键作用, 在过渡金属催化下对特定位置的碳氢 键进行活化(Scheme 1c).

(a) Conventional directing group strategy

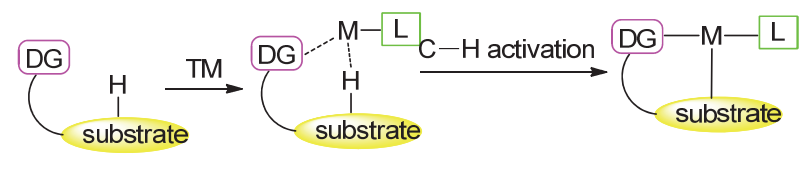

(b) Transient directing group strategy

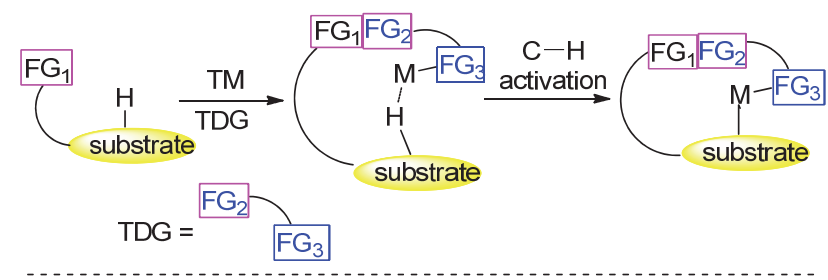

(c) Noncovalent interaction strategy

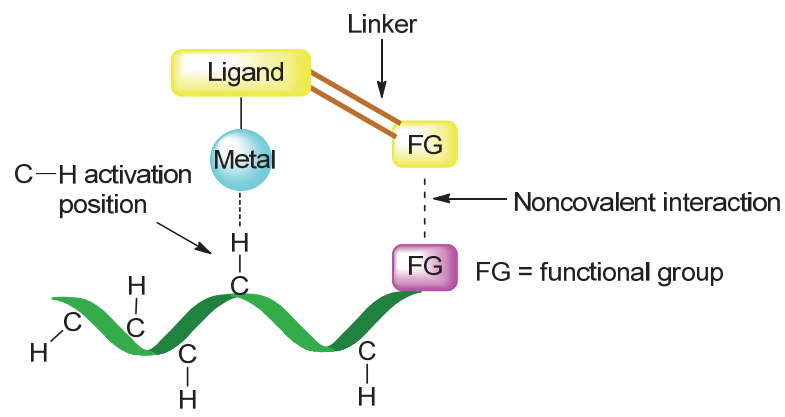

图式 1 位点选择性碳氢键官能团化策略

Scheme 1 Diverse strategy for site-selective $\mathrm{C}-\mathrm{H}$ functionalization

对于非共价键参与的位点选择性碳氢键活化, 常常 受到如下三个因素的影响: (1)底物的官能团需要和配体 的官能团发生非共价作用, 使得过渡金属能够对特定位 点的碳氢键进行活化; (2)过渡态的稳定性; (3)反应体系 对非共价作用的兼容性. 接下来将按照非共价键的作用 类型来分, 主要有氢键作用、离子对作用、路易斯酸碱 对作用、静电作用等.

\section{2 不同类型的非共价作用}

\section{1 氢键作用}

2012 年, Singleton 等 ${ }^{[8]}$ 报道了铱催化叔丁氧羰基 (Boc)保护的苯胺邻位碳氢键硼化反应(Scheme 2). 在该
反应中, 硼化选择性地发生在苯胺的邻位. 通过实验和 理论计算研究表明, 这种选择性主要是由于底物分子中 氮上的氢原子与嗍酸频哪醇酯中的氧原子形成了氢键 (TS1). 在铱催化剂的作用下, 对苯胺邻位碳氢键进行 活化，实现高选择性嗍化. 该反应能够兼容间位和对位 具有取代基的叔丁氧羰基保护的苯胺，而对于邻位具有 取代基的底物作者未给出例子. 之后，该硼化反应很顺 利地拓展到无保护的苯胺底物上 ${ }^{[9]}$. 相对于之前的喼酸 频哪醇酯作为硼基的主要来源, 作者采用频哪醇硼烷作 为嗍基来源，与苯胺原位形成中间体(TS2), 之后通过 之前类似的机理实现苯胺的邻位硼化.

Singleton, Smith, Maleczka, et al., 2012

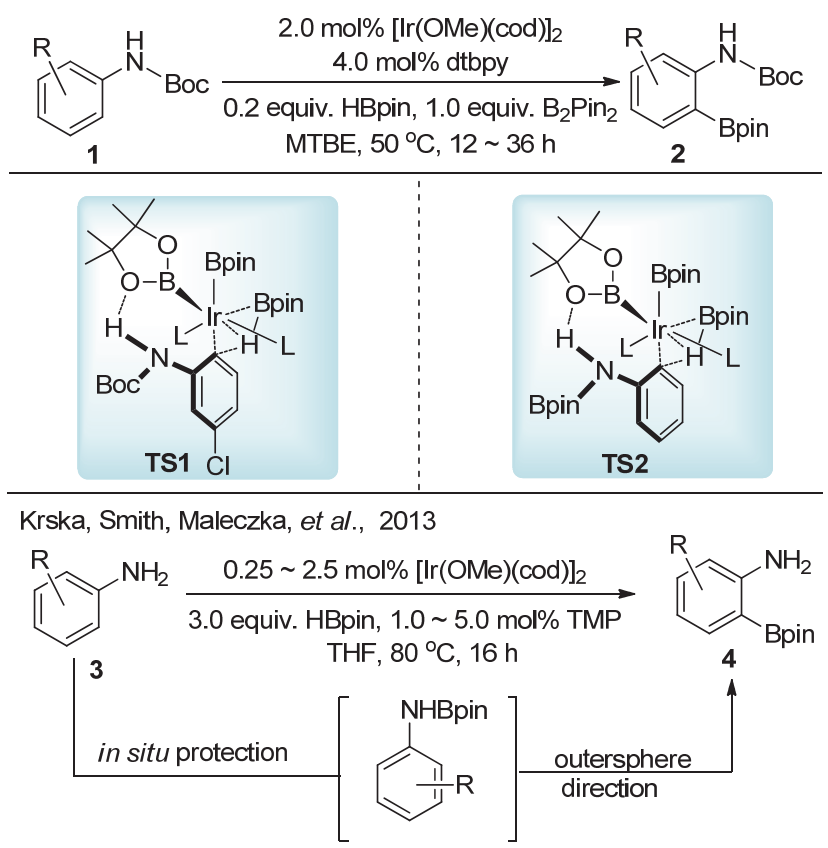

图式 2 叔丁氧羰基(Boc)保护苯胺及苯胺的邻位选择性碳氢键嗍化 Scheme 2 otho-Selective $\mathrm{C}-\mathrm{H}$ borylation of $N$-Boc anilines and free anilines

2015 年, Kanai 和 Kuninobu 课题组 ${ }^{[10]}$ 报道了铱催化 的苯甲酰胺间位选择性碳氢键硼化反应(Scheme 3). 他 们的间位选择性㸴化策略也是基于氢键，对于配体的设 计有着较高的要求. 他们认为, 反应基于 TS3 反应模型. 首先, 苯甲酰胺的羰基氧与配体中的艮形成氢键, 而后 过渡金属铱与配体分子中的联吡啶结构进行配位，进而 对底物分子的间位或者对位碳氢键进行活化. 通过对配 体分子结构的有效调节，最终配体 L1 更有利于间位碳 氢键活化. 该反应底物适用范围广，各种取代的苯甲酰 氨、杂环和苯基磷酸酯等都能很好兼容，能够得到选择 性较高的间位硼化产物. 在这之后, Kuninobu 课题组 ${ }^{[11]}$ 进一步利用这一策略, 将底物进行间位硼化后, 未经分 离而进一步将其转化成其他官能团，包括溴、氯、羟基、 苯硫基、叠氮、氰基、芳基、烯基、烷基和烯丙基等. 
Kanai \& Kuninobu, 2015
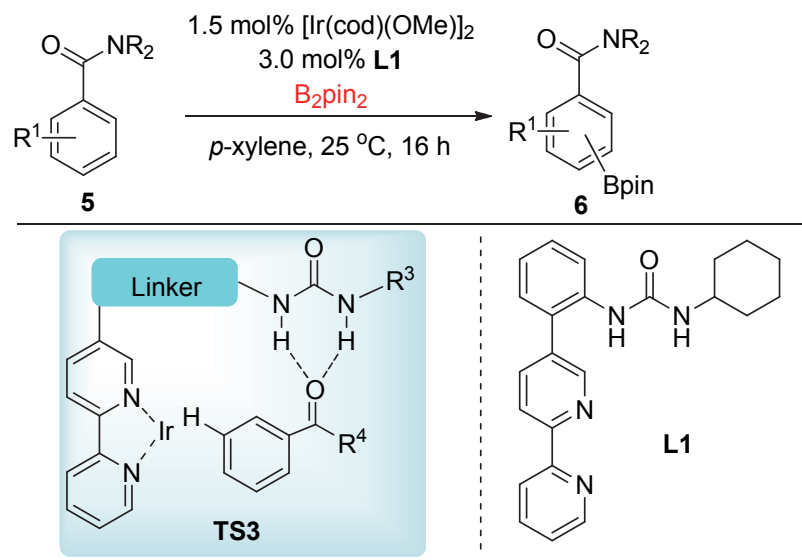

selected examples
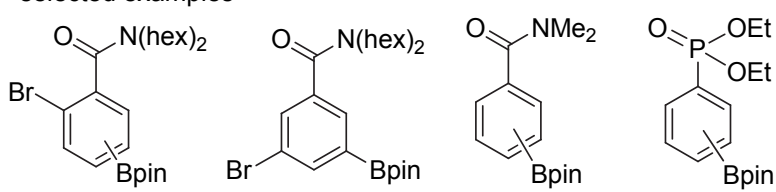

6a, $96 \%$,

6b, $85 \%$

6c, $44 \%$,

6d, $52 \%$,

$m: p=7.5: 1$

Kuninobu, 2019<smiles>[R]NC(=O)c1[R1]cccc1</smiles>

(1) cat. $[\operatorname{Ir}(\mathrm{OMe})(\mathrm{cod})]_{2}$
L1, $\mathrm{B}_{2} \mathrm{pin}_{2}$
(2) functionalizaiton

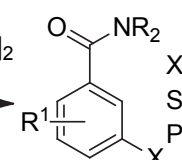

$\mathrm{X}=\mathrm{Cl}, \mathrm{Br}, \mathrm{OH}$ $\mathrm{SPh}, \mathrm{N}_{3}, \mathrm{NH}_{2}, \mathrm{CN}$, $X^{\mathrm{Ph} \text {, Pyridine, etc. }}$

5

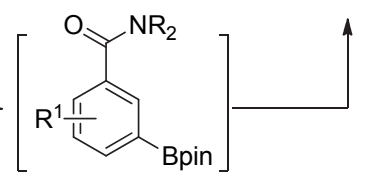

图式 3 氢键控制的苯甲酰胺间位碳氢键硼化及其转化

Scheme 3 Hydrogen-bonding-enabled meta-selective $\mathrm{C}-\mathrm{H}$ borylation and transformations of benzamides

Kuninobu 和 Kanai 课题组 ${ }^{[12]}$ 对基于氢键策略的芳 环化合物间位选择性碳氢键嗍化反应中含䐂结构的联 吡啶配体进行了深入的探究(Scheme 4). 他们发现, 改 变 $R^{1}$ 位点能够改变配体的催化活性; 调节 $R^{2}$ 位点能够 调整反应的过渡态, 而调节 $\mathrm{R}^{3}$ 位点则能够改变䐂结构 与羰基形成氢键的能力. 这一系统性的研究有望为未来 配体设计提供初步的结构-活性关系. Sunoj 课题组 ${ }^{[13]}$

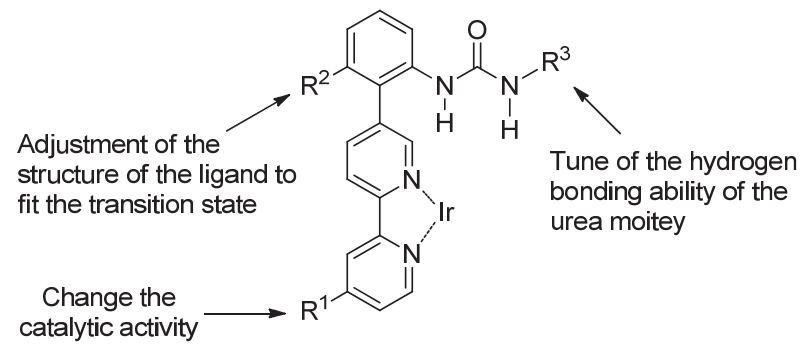

图式 4 氢键控制的间位选择性碳氢键硼化的配体

Scheme 4 Relationship between ligand structure and reactivity for hydrogen-bonding-enabled meta-selective $\mathrm{C}-\mathrm{H}$ boryaltion
通过密度泛函理论(DFT)对该反应的机理进行了研究, 他们发现硫嫝结构的酰胺氢对反应选择性有很大影响, 该氢原子能够与底物中的羰基形成氢键作用, 在能量上 更加趋向于活化底物间位. 并且底物 $6 \mathrm{c}$ 与含有配体 $\mathbf{L 1}$ 的催化剂铱之间的非共价作用使得间位和对位活化时 能量有所差异，间位碳氢键活化的过渡态在能量上比对 位要低 $27.6 \mathrm{~kJ} \cdot \mathrm{mol}^{-1}$, 而这个能量差值可以使间位和对 位的选择性达到 $27: 1$.

2017 年, Phipps 等 ${ }^{[14]}$ 报道了茮胺衍生物的间位碳氢 键硼化反应(Scheme 5). 他们利用联吡啶磺酸盐中的磺
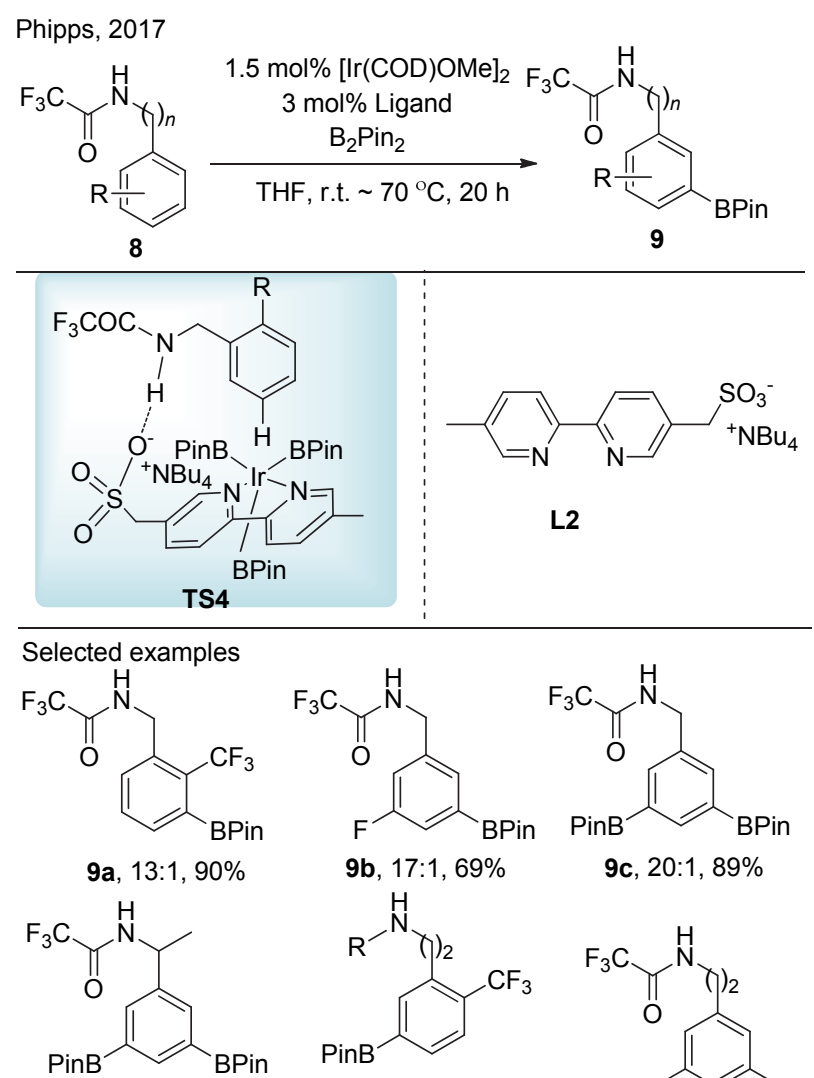

9d, $11: 1,41 \%$

9e, $\mathrm{R}=\mathrm{TFA}, 18: 1,86 \%$ 9f, $R=M s, 20: 1,93 \%$

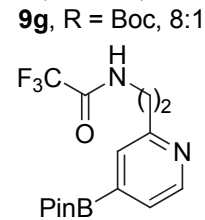

9c, $20: 1,89 \%$<smiles>O=C(Nc1cc(Pc2ccccc2)ccc1C(F)(F)F)C(F)(F)F</smiles>

PinB<smiles>O=C(NCc1cc(F)cc(P)c1)C(F)(F)F</smiles>

9h, 13:1, 89\%<smiles>CCN(Cc1cc(Pc2ccccc2)ccc1C(F)(F)F)C(=O)C(F)(F)F</smiles>

9i, $20: 1,97 \%$

9j, 5:1, 94\%

9k, $2.4: 1 m: p, 71 \%$

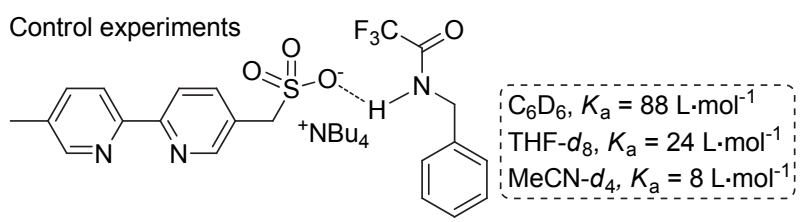

图式 5 氢键控制的茮胺、苯乙胺、苯丙胺衍生的三氟乙酰胺间位碳 氢键嗍化

Scheme 5 Hydrogen-bonding-enabled meta-C $-\mathrm{H}$ borylation of benzylamine-, phenethylamine- and phenylpropylamine-derived trifluoroacetamides 
酸根与底物分子的酰胺氢形成氢键作用, 实现了间位碳 氢键的选择性硼化. 该反应能够兼容一系列三氟乙酰基 保护的苄胺、苯乙胺和苯丙胺, 对于吡啶杂环也有较好 的兼容性. 为了验证底物与配体之间存在氢键作用, 他 们进行了一系列对照实验. 首先, 在不同溶剂中, 核磁 滴定表明底物分子和配体间存在相互作用, 即使在极性 溶剂乙腈中其作用也非常明显. 其次, 当底物的酰胺氢 被取代后，其间位硼化和对位嗍化产物的比例显著降 低, 说明配体与底物之间的氢键作用能够影响反应的选 择性. 值得一提的是, 该类磺酸根配体最初由 Phipps 课 题组 ${ }^{[15]}$ 设计用于离子对作用的碳氢键活化 (见 2.2 节).

铱催化芳香酰胺的碳氢键硼化反应, 由于电子效应 的影响, 其邻位碳氢健的活化是不利的. 为克服这一困 难, Reek 课题组 ${ }^{[16]}$ 报道了氢键控制的铱催化芳香酰胺邻 位选择性碳氢键硼化反应(Scheme 6). 该反应采用二级 酰胺作为底物, 设计了含有吲哚结构的联吡啶骨架配体 L3, 该配体的吲哚氮氢和酰胺氮氢能够与底物酰胺的 氧形成氢键, 从而通过过渡态 TS5 实现铱催化酰胺的邻 位碳氢键的活化，最终高选择性地得到邻位嗍化产物.

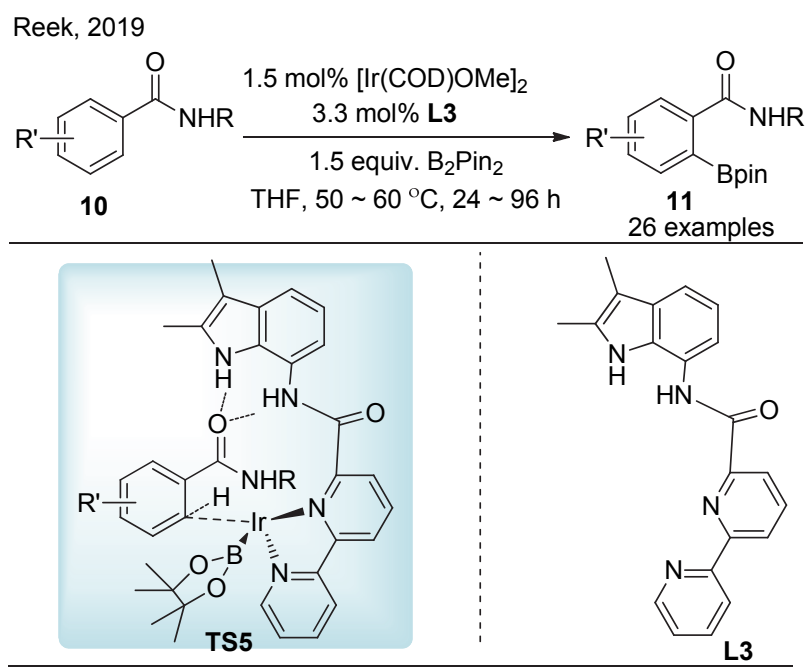

图式 6 氢键控制的芳香酰胺的邻位碳氢键嗍化

Scheme 6 Hydrogen-bonding-enabled ortho-selective C $-\mathrm{H}$ borylation of secondary aromatic amides

\section{2 离子对作用}

2016 年, Phipps 课题组 ${ }^{[15]}$ 报道了一种全新的非共价 键策略用于铱催化间位碳氢键硼化反应(Scheme 7). 该 反应中底物为季铵盐, 配体为具有磺酸根的联吡啶. 通 过离子交换, 底物的阳离子与配体的磺酸根离子产生离 子对作用, 配体中联吡啶与铱催化剂配位, 进而选择性 活化底物分子中的间位碳氢键. 一系列的苠胺、苯胺和 含杂环的季铵盐均能够高选择性地发生间位碳氢键硼 化. 为体现该反应的应用价值, 作者通过偶联反应将季 铵盐进一步转化为联芳化合物. 在这之后, 该课题组进 一步利用该策略, 将底物范围拓展到苯乙胺和苯丙胺的
季铵盐衍生物之中 ${ }^{[17]}$. 在这些工作的基础上, Phipps 课 题组 ${ }^{[18]}$ 继续将该方法用于芳基膦盐的硼化之中.

Phipps, 2016
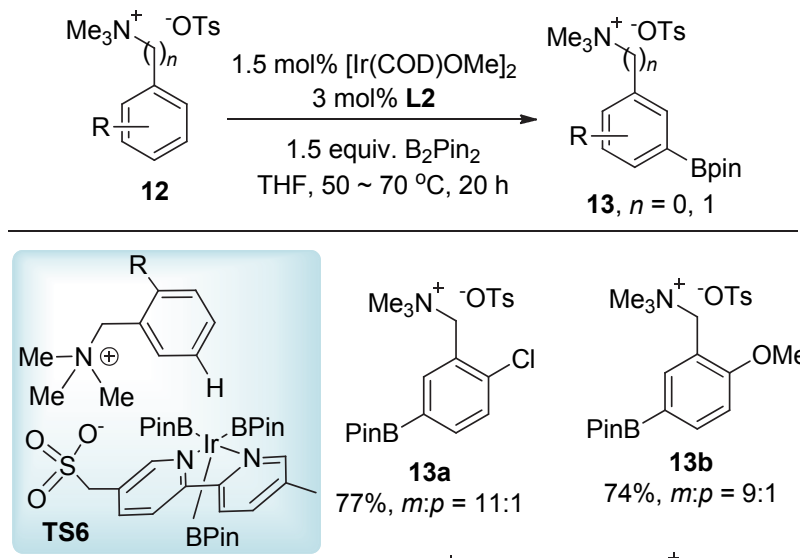

$77 \%, m: p=11: 1$

$74 \%, m: p=9: 1$
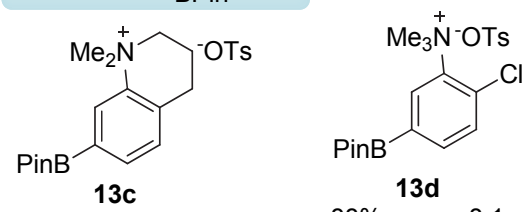

$13 d$

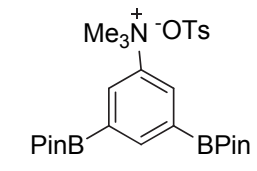

$80 \%, m: p \geqslant 20: 1$

$88 \%, m: p=8: 1$

$80 \%, m: p \geqslant 20: 1$

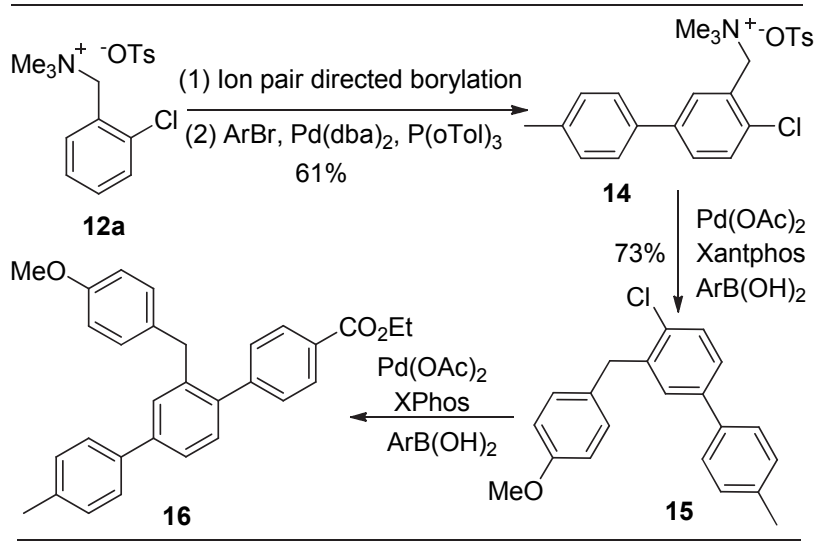

Phipps, 2018

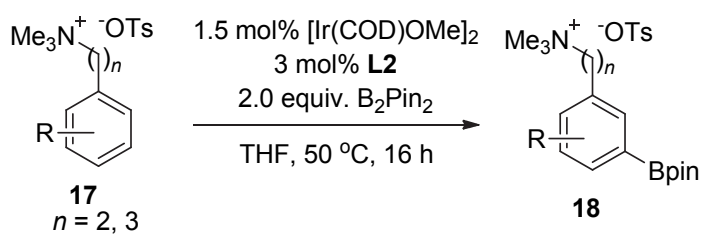

Phipps, 2019
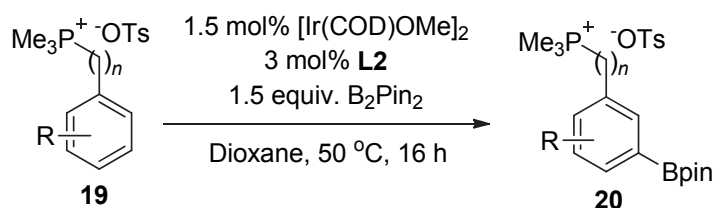

$n=0,1,2$

20

图式 7 离子对控制的季胺盐间位碳氢键硼化反应

Scheme 7 Ion pair-enabled meta-C $-\mathrm{H}$ borylation of quaternary ammonium salts

最近, Phipps 课题组 ${ }^{[19 a]}$, Maleczka 和 Smith 课题 组 ${ }^{[19 b]}$ 分别报道了基于离子对策略的铱催化对位选择性 碳氢键硽化(Scheme 8). 在该反应中，底物(苯酚、苄醇、 


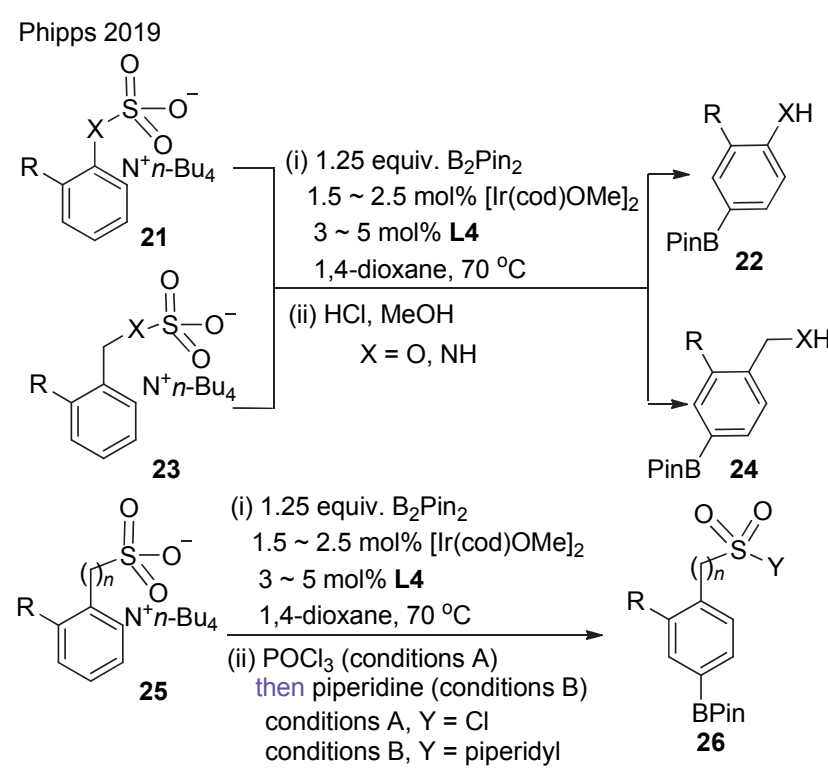

Maleczka, Smith, 2019

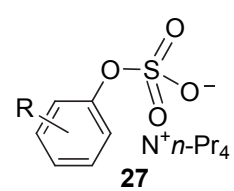

(i) 1.25 equiv. $\mathrm{B}_{2} \mathrm{Pin}_{2}$

$1.5 \mathrm{~mol} \%[\operatorname{lr}(\mathrm{cod}) \mathrm{OMe}]_{2}$

(ii) $12 \mathrm{~mol} / \mathrm{L} \mathrm{HCl}, \mathrm{pH}=1 \sim 2,1 \mathrm{~h}$

(i) 1.25 equiv. $\mathrm{B}_{2} \mathrm{Pin}_{2}$<smiles>[R]c1cccc(NS(=O)(=O)[O-])c1</smiles>

$3.0 \mathrm{~mol} \%[\operatorname{lr}(\mathrm{cod}) \mathrm{OMe}]_{2}$

$6.0 \mathrm{~mol} \%$ L5

1,4-dioxane, $60{ }^{\circ} \mathrm{C}, 10 \sim 36 \mathrm{~h}$

(ii) $12 \mathrm{~mol} / \mathrm{L} \mathrm{HCl}, \mathrm{pH}=1 \sim 2,1 \mathrm{~h}_{\mathrm{Pin}}$

(i) 1.25 equiv. $\mathrm{B}_{2} \mathrm{Pin}_{2}$

$1.5 \mathrm{~mol} \%[\operatorname{lr}(\mathrm{cod}) \mathrm{OMe}]_{2}$

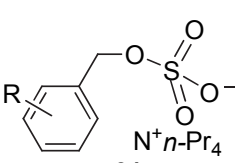

31

$3.0 \mathrm{~mol} \%$ L5

1,4-dioxane, $40^{\circ} \mathrm{C}, 12 \sim 20 \mathrm{~h}$

(ii) $12 \mathrm{~mol} / \mathrm{L} \mathrm{HCl}, \mathrm{pH}=1 \sim 2,1 \mathrm{~h}$

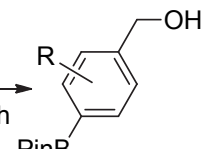

32

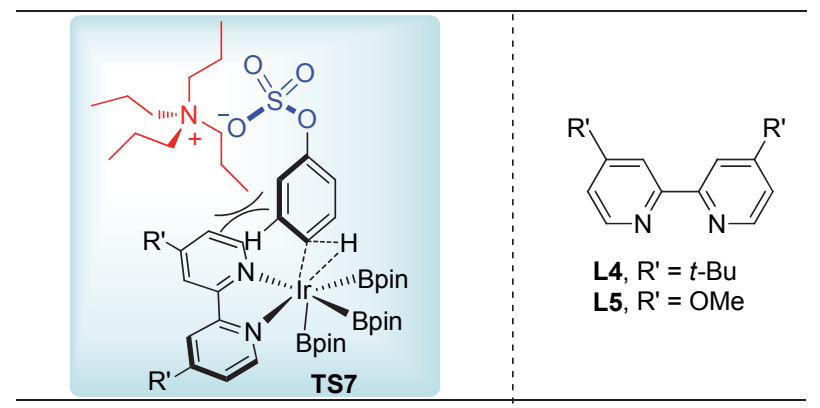

Position selectivity vs the cation size
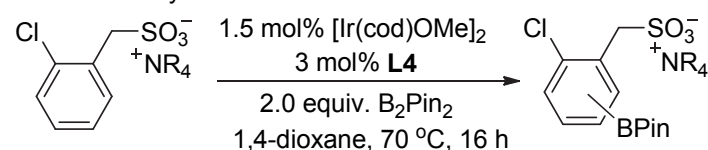

$\mathrm{Me}_{4} \mathrm{~N}^{+} \quad \mathrm{Et}_{4} \mathrm{~N}^{+}$

1,4-dioxane, $70^{\circ} \mathrm{C}, 16 \mathrm{~h}$

$3.5: 1 \quad 4: 1$

\begin{abstract}
$\mathrm{Pr}_{4} \mathrm{~N}^{+}$
\end{abstract}
$\mathrm{Bu}_{4} \mathrm{X}^{+}$

$\mathrm{Hex}_{4} \mathrm{~N}^{+}$

8.6:1

$X=N, 10: 1$

p:m selectivity

13:1
图式 8 铱催化苯酚、苄醇和苯胺的对位碳氢键硼化

Scheme 8 Ir-catalyzed para-C $-\mathrm{H}$ borylation of phenols, benzyl alcohols and anilines
苯胺或芐胺)先转化成相应的硫酸盐或磺酰盐, 其阳离 子部分为四烷基(丙基或者丁基)季铵盐，利用该季铵盐 的大位阻效应阻止底物间位碳氢键金属化，从而高选择 性地实现了对位碳氢键嗍化. 嗍化之后的铵盐在浓盐酸 作用下水解得到对位硼化的苯酚、苯胺或茮醇产物. Phipps 等以 2-氯芐基磺酸盐为底物, 对阳离子大小与对 /间位选择性进行了系统研究. 实验表明, 对位选择性随 着阳离子的增大而明显增加.

\section{3 路易斯酸碱对作用}

2016 年, Chattopadhyay 课题组 ${ }^{[20]}$ 报道了非共价作 用促进的铱催化芳香醛间位选择性碳氢键硼化 (Scheme 9). 该反应通过芳基醛与烷基胺反应现场生成亚胺，亚 胺与铱催化剂上的频哪醇硼通过非共价作用, 诱导实现 铱催化的间位碳氢键嗍化，3,4,7,8-四甲基-1,10-邻菲咯 Chattopadhyay, 2016

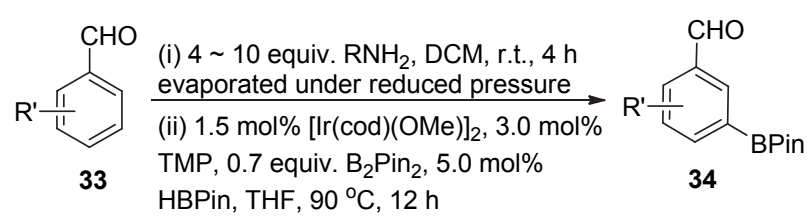

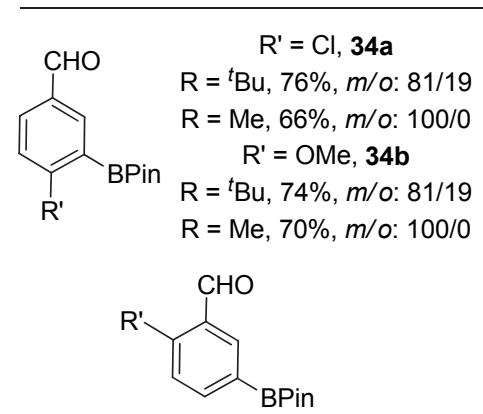

$\mathrm{R}^{\prime}=\mathrm{Cl}, \mathbf{3 4 g}$

$\mathrm{R}={ }^{t} \mathrm{Bu}, 65 \%, \mathrm{~m} / \mathrm{o:} 70 / 30$

$\mathrm{R}=\mathrm{Me}, 73 \%, \mathrm{~m} / \mathrm{o}: 100 / 0$ $\mathrm{R}^{\prime}=\mathrm{Br}, \mathbf{3 4 h}$

$\mathrm{R}={ }^{t} \mathrm{Bu}, 69 \%, \mathrm{~m} / \mathrm{o}: 85 / 15$

$\mathrm{R}=\mathrm{Me}, 63 \%, \mathrm{~m} / \mathrm{o}: 100 / 0$

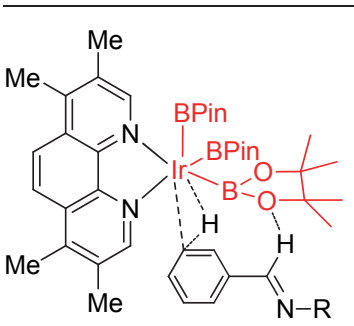

TS8: hydrogen-bonding

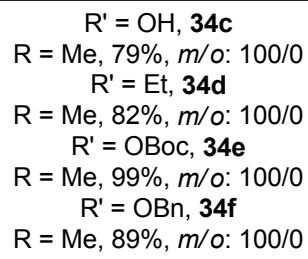
$\mathrm{R}^{\prime}=\mathrm{OH}, \mathbf{3 4 c}$

$\mathrm{R}=\mathrm{Me}, 79 \%, \mathrm{~m} / \mathrm{o}: 100 / 0$ $\mathrm{R}^{\prime}=\mathrm{Et}, \mathbf{3 4 d}$

$\mathrm{R}=\mathrm{Me}, 82 \%, \mathrm{~m} / \mathrm{o}: 100 / 0$ $\mathrm{R}^{\prime}=\mathrm{OBoc}, 34 \mathrm{e}$

$\mathrm{R}=\mathrm{Me}, 99 \%, \mathrm{~m} / \mathrm{o}: 100 / 0$ $\mathrm{R}^{\prime}=\mathrm{OBn}, \mathbf{3 4 f}$

$\mathrm{R}=\mathrm{Me}, 89 \%, \mathrm{~m} / \mathrm{o}: 100 / 0$

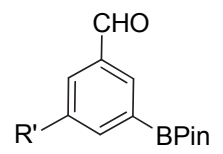

$\mathrm{R}^{\prime}=\mathrm{Cl}, \mathbf{3 4 i}$

$\mathrm{R}={ }^{t} \mathrm{Bu}, 93 \%, m / o+p: 100 / 0$

$\mathrm{R}^{\prime}=\mathrm{Br}, \mathbf{3 4} \mathbf{j}$

$\mathrm{R}={ }^{t} \mathrm{Bu}, 98 \%, m / o+p: 100 / 0$

$\mathrm{R}^{\prime}=\mathrm{CN}, \mathbf{3 4 k}$

$\mathrm{R}={ }^{t} \mathrm{Bu}, 91 \%, m / o+p: 100 / 0$

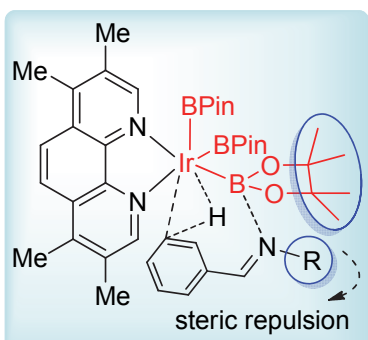

TS9: B-N Lewis pair

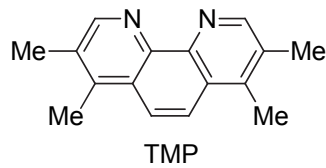

图式 9 Iridium-catalyzed meta-selective $\mathrm{C}-\mathrm{H}$ borylation of aromatic aldehydes

Scheme 9 铱催化芳香醛间位选择性碳氢键硼化 
啉(TMP)作为配体调控催化中心金属的电性. 他们认 为, 反应的选择性可能是来源于 TS8 中的氢键作用, 或 者是 TS9 中氮一嗍的路易斯酸碱对作用. 作者认为, 在 TS8 中, 亚胺的取代基远离反应中心, 因此选择性应该 不受烷基胺上取代烷基(R)的大小影响; 而与此相反, 在 TS9 中, 由于烷基胺的取代基(R)与频哪醇的位阻排 斥, 位阻更小的 $\mathrm{R}$ 基团更加有利于反应. 基于以上设想 以及反应中甲胺给出比叔丁胺更好的反应选择性, 作者 倾向于认为该反应的选择性是基于路易斯酸碱对作用 TS9. 这一工作虽然不是通过设计的配体与底物的路易 斯酸碱对作用, 但也为后续工作提供了有益的参考.

2017 年, Kuninobu 和 Kanai 课题组 ${ }^{[21]}$ 报道了芳香硫 醚的邻位嗍化反应. 该反应中底物的硫原子与配体的硼 原子之间形成路易斯酸碱对作用(Scheme 10, TS10). 他 们通过研究发现, 通过配体结构改变能够调控反应的选 择性, 当 4,4-二一叔丁基联吡啶(dtbpy)作为配体时其邻 位选择性较差. 对联吡啶进一步改造, 当采用配体 $\mathbf{L 4}$ 类型配体时, 邻位选择性显著增加. 配体上的嗍保护基 的电性对反应的活性和邻位的选择性也有非常大的影 响. 在硼的保护基团中引入三氟甲基, 其产率和邻位选 择性均显著提高 $(64 \%, o / m+p>30)$. 一系列的 3 位或者 4 位取代的芳基硫醚均能够以较高的邻位选择性得到间 位硼化产物, 含有吡啶、呋喃和吡咯等的杂芳环硫醚则 难以适用于该体系, 可能是杂芳环本身杂原子与嗍原子 的路易斯酸碱对作用的影响.

Kuninobu and Kanai, 2017

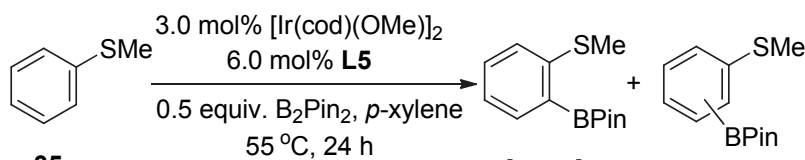

35 $55^{\circ} \mathrm{C}, 24 \mathrm{~h}$

[ortho]

$[$ meta + para]
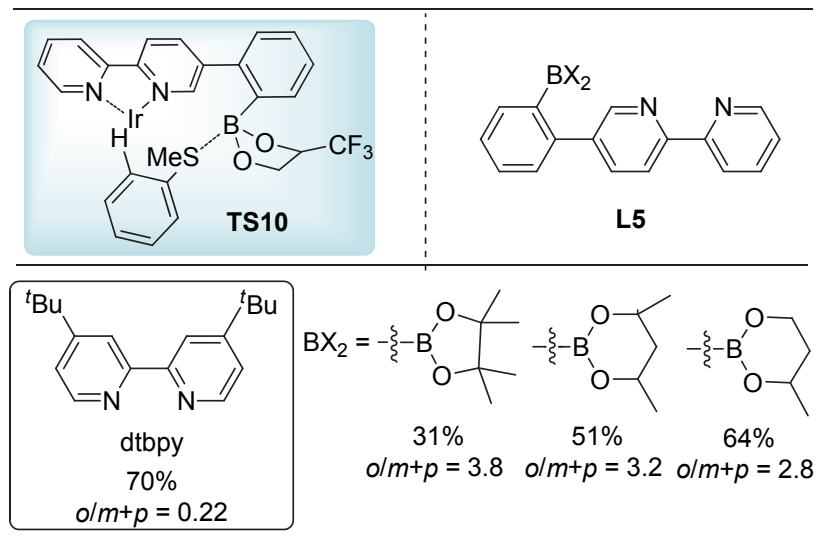<smiles>COOC[C@H]1OB([Te])O[C@@H]1C(=O)OC</smiles>

$55 \%$

$o / m+p=2.3$ $<1 \%$

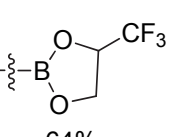

$64 \%$

$\mathrm{o} / m+p>30$

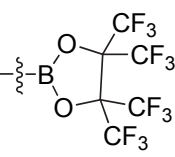

$<1 \%$
图式 10 路易斯酸碱对控制的芳烃硫醚的邻位选择性碳氢键硼化

Scheme 10 Lewis acid-base interaction-enabled ortho-selective $\mathrm{C}-\mathrm{H}$ borylation of aryl sulfides
2017 年, Nakao 课题组 ${ }^{[22]}$ 报道了芳香化合物的对位 选择性碳氢键硼化反应(Scheme 11). 该反应中，底物上 的路易斯碱官能团和路易斯酸形成酸碱对，通过电性传 递使得底物的芳环更加缺电子，从而提高反应活性 (TS11). 同时，配位于铱上的配体与大位阻的铝之间产 生排斥作用，在空间上阻碍了金属铱对邻位和间位碳氢 键的活化，从而实现了对位碳氢键的高选择性活化. 该 反应的底物范围广，对于苯甲酰胺、芳基磷酸酯以及吡 啶等杂环都有着良好的兼容性.

Nakao, 2017
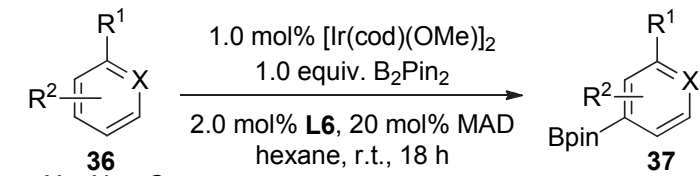

$\mathrm{X}=\stackrel{36}{\mathrm{~N}}$ or $\mathrm{C}$
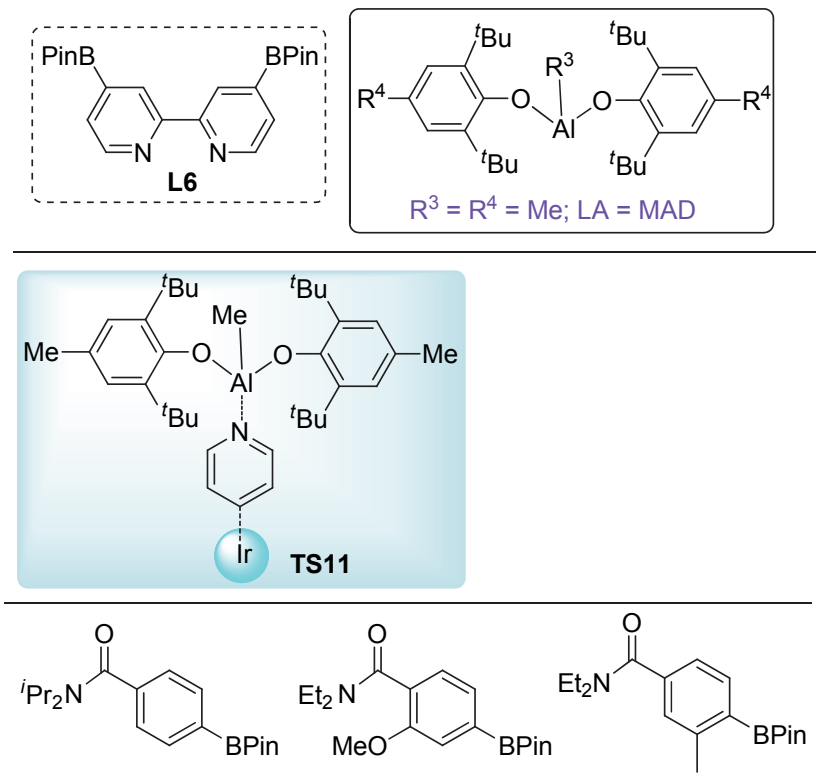

37a, 95\%, $p: m>20: 1$

37b, 95\%, $p: m>20: 1$

37c, $55 \%, p: m 0: 1$<smiles>CCOP(=O)(OCC)c1ccc(Br)cc1Br</smiles>

37d, 92\%, p:m 6.6:1<smiles>CCNC(=O)c1ccc(Br)cn1</smiles>

37e, $74 \%, p: m>20: 1$<smiles>CCNC(=O)c1ccc(Br)n1C</smiles><smiles>CCNC(=O)c1ccc(Br)s1</smiles>

$37 f, 90 \%$ 37g, 100\%, p:m 7.3:1

图式 11 铱/铝催化的芳环对位选择性碳氢键硼化

Scheme 11 para-Selective $\mathrm{C}-\mathrm{H}$ borylation of (hetero)arenes by cooperative iridium/aluminum catalysis

2019 年, Nakao 课题组 ${ }^{[23]}$ 将该铱铝双功能催化剂应 用于芳香化合物间位碳氢键的选择性嗍化(Scheme 12). 其催化剂结构主要包括可用于与铱配位的联吡啶或菲 咯啉结构和联吡啶与铝的连接部分. 具有路易斯酸性的 烷基铝能够识别酰胺的羰基或者 $\mathrm{sp}^{2}$ 杂化的氮. 通过系 
统的研究, 他们发现具有烷基铝联苯氧化物的 2,2'-联吡 啶配体 L7，在铱催化下能实现苯甲酰胺的高选择性间 位碳氢键硼化反应. 当使用 1,10-菲咯啉配体(L8)时, 可 以实现吡啶 C5 (C3)位选择性碳氢键硼化.

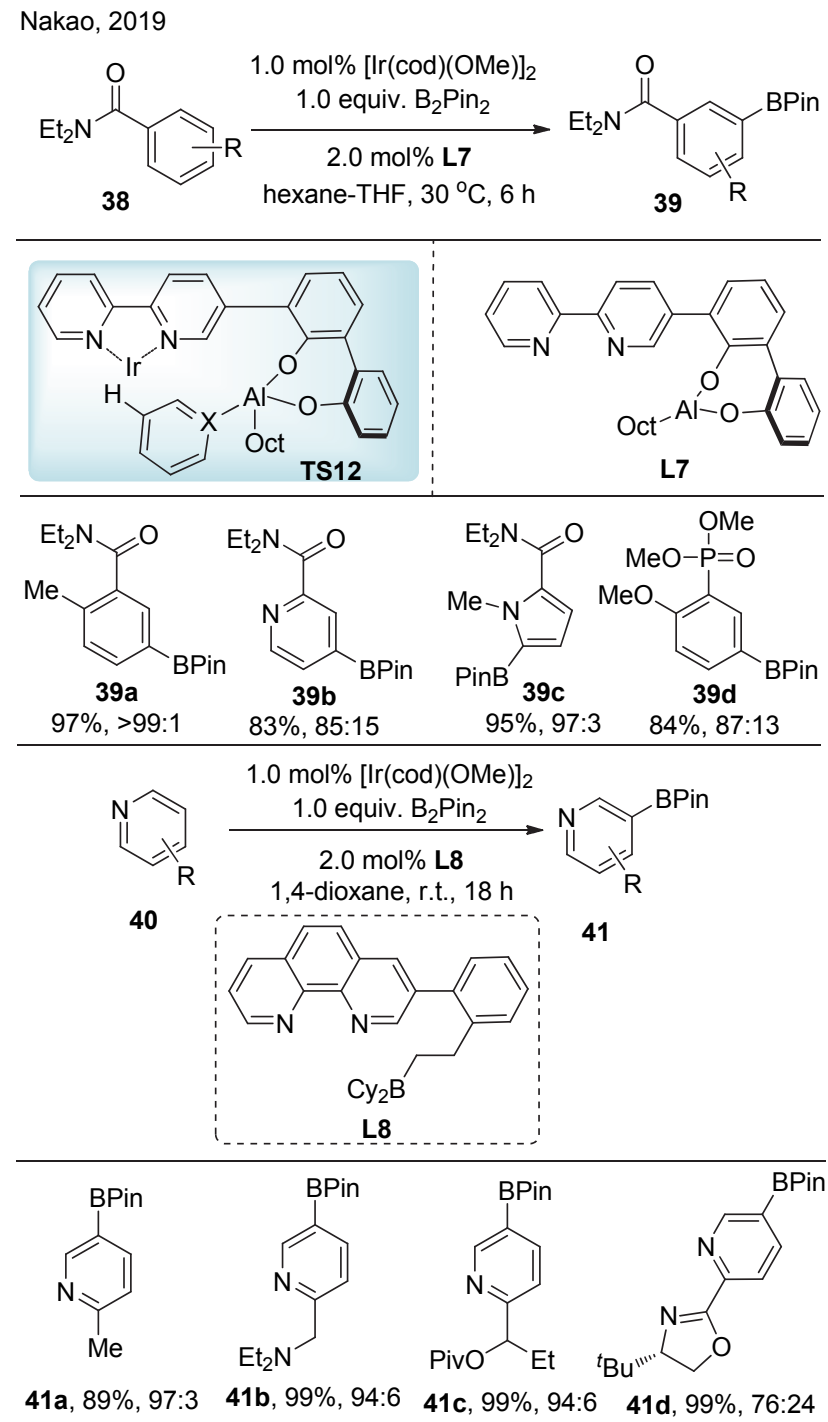

图式 12 铱/铝催化的苯甲酰胺及吡啶的间位选择性碳氢键硼化

Scheme 12 meta-Selective $\mathrm{C}-\mathrm{H}$ borylation of benzamides and pyridines by an iridium-aluminum bifunctional catalyst

2017 年, Chattopadhyay 课题组 ${ }^{[24]}$ 报道了铱催化芳 香酯选择性对位碳氢键硼化反应(Scheme 13). 该反应 采用 8 位连接 2,2-联吡啶的喹诺酮配体模板 L9. 作者认 为, L9 的喹诺酮单元在反应中可以互变异构为 2-差基喹 啉, 与反应体系中的叔丁醇钾反应, 形成 $\mathbf{L 9}$ 的钾盐, 钾 离子与底物的酯羰基形成路易斯酸碱对, 通过如 Scheme 13 所示过渡态 TS13, 实现对位碳氢键硼化. 该 反应能够选择性活化芳香酯的对位, 对于杂环底物也具 有较好的位置选择性. 为验证该模型的可能性, 作者进 行了几组对照实验. 首先, 当采用 $\mathbf{L 1 0}$ 作为配体时, 其 对位与间位的选择性达到 33/1. 将配体用甲基保护后得
到 L11, 其对位与间位选择性仅为 1.9/1. 再次，作者以 L9 为配体，在体系中加入 18-冠-6 醚，当体系中没有叔 丁醇钾存在时, 其对位与间位选择性为 5.6/1; 当体系中 具有叔丁醇钾时，由于 18 -冠-6 醚捕获钾离子，其对位 与间位选择性由 $33 / 1$ 降低为 $1.8 / 1.0$, 该结果进一步验证 了钾离子对反应的选择性有着较大的影响, 证明体系中 可能存在钾离子与酯羰基的弱相互作用.

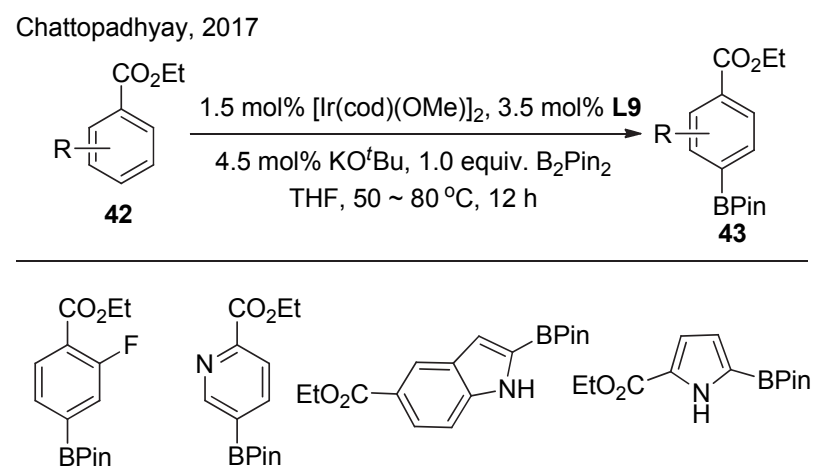

43a, $91 \% \quad 43 b, 77 \% \quad$ 43c, $40 \% \quad 43 d, 81 \%$ p/other $=28 / 1$ p/other $=8: 1 \quad C^{2} /$ other $=99 / 1 \quad C^{2} /$ other $=99 / 1$
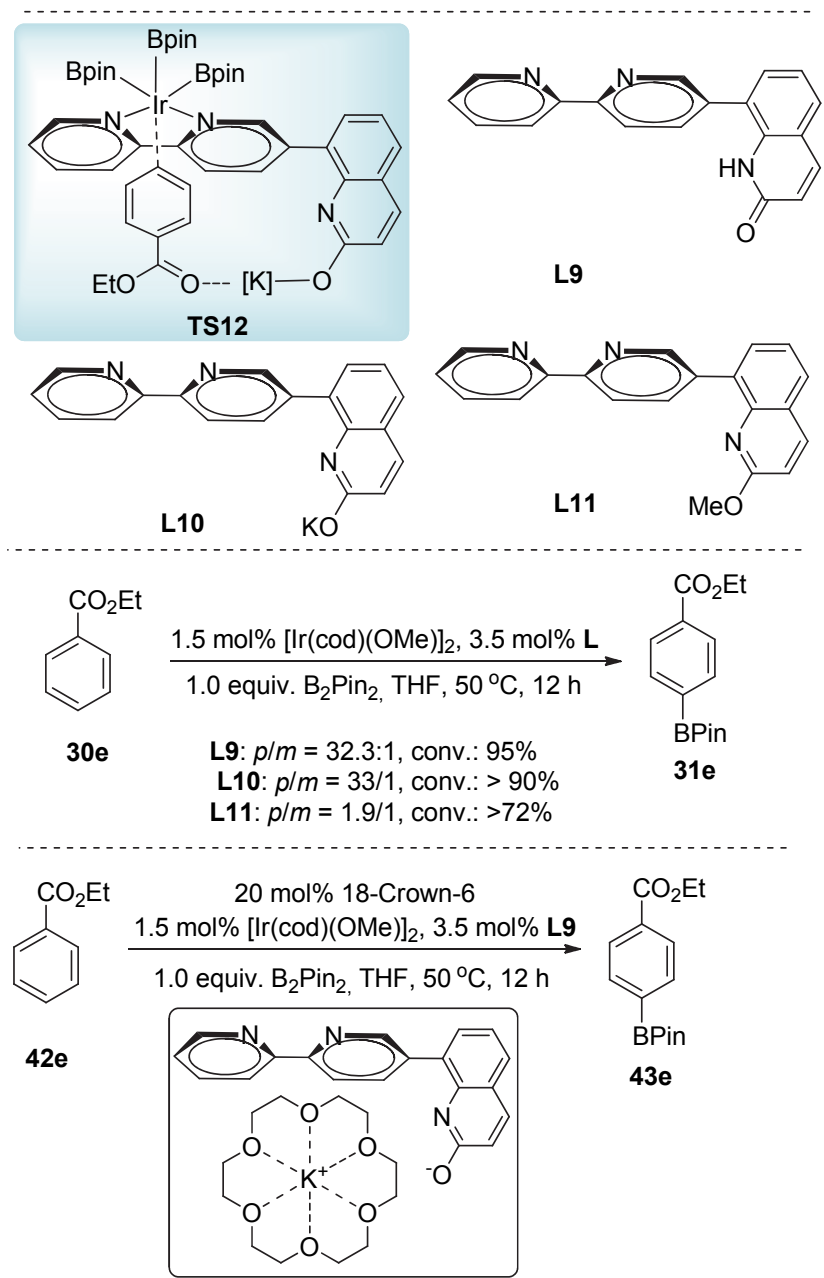

with $4.5 \mathrm{~mol} \% \mathrm{KO}{ }^{t} \mathrm{Bu} p / m=1.8 / 1.0$; conv. $59 \%$ without $\mathrm{KO}{ }^{t} \mathrm{Bu} p / m=5.6 / 1.0$; conv. $90 \%$

图式 13 铱催化芳香酯对位碳氢键硼化

Scheme 13 Iridium-catalyzed para-C $-\mathrm{H}$ borylation of aromatic esters 
Chattopadhyay 课题组 ${ }^{[25]}$ 随后将配体 $\mathbf{L 9}$ 应用于铱催 化的芳香酰胺类化合物间位碳氢键的选择性硼化 (Scheme 14). 该反应能够兼容吡啶、呋喃、噻吩和吲哚 等杂环化合物. 当用 $\mathbf{L 1 0}$ 作为配体时, 其对位与间位的 选择性为 28/1; 当用 $\mathbf{L 1 1}$ 作为配体时, 其间位与对位的 选择性为 $1.1 / 1$; 将底物变为茮胺, 其选择性急剧下降. 以上对照实验表明, 与之前反应类似, 体系中存在钾离 子与底物酰胺的非共价键作用. 他们推测可能有两种作 用方式: 酰胺羰基与钾离子的路易斯酸碱作用(TS14)或 者离子- $\pi$ 作用(TS15). 作者认为, 经由 TS15 的离子- $\pi$ 作用将显著受到底物电性和反应溶剂极性的影响. 对照 实验表明, 无论是给电子 $(44 a, M e O)$ 还是缺电子底物 $\left(44 b, C F_{3}\right)$ 都给出相当的反应活性和选择性; 另外, 反应 溶剂换成非极性溶剂正己烷，产率略有下降，但选择性 几乎相同. 因此, 虽然无法完全排除离子- $\pi$ 作用, 但作 Chattopadhyay, 2018
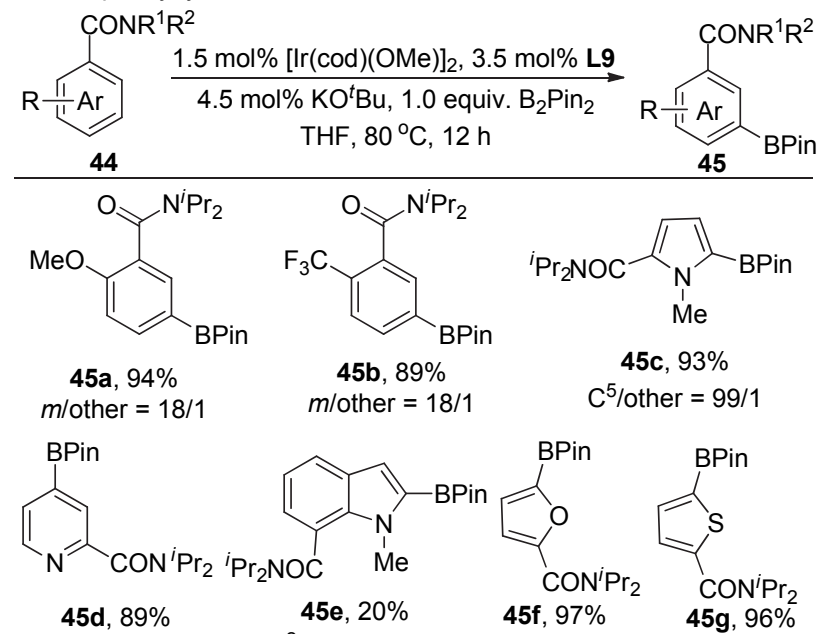
m/others $=18 / 1 \quad$ C $^{2} /$ other $=99 / 1 \quad$ C $^{5} /$ other $=99 / 1 \quad$ C $^{5} /$ other $=99 / 1$
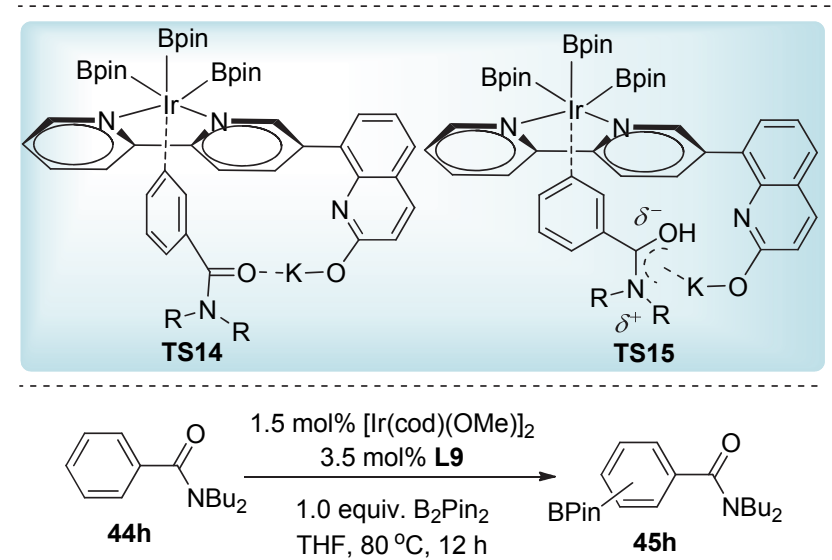

L10: $p / m=28 / 1$, cov.: $>77 \%$; L11 $m / p=1.1 / 1$, cov.: $>90 \%$

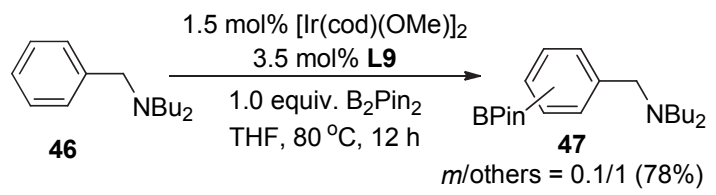

图式 14 铱催化芳香酰胺的间位碳氢键嗍化

Scheme 14 Ir-catalyzed meta-C $-\mathrm{H}$ borylation of aromatic amides
者更倾向于反应是经由路易斯酸碱对作用.

\section{4 静电作用}

2017 年, Maleczka 等 ${ }^{[26]}$ 报道了铱催化苯酚邻位的硼 化反应(Scheme 15). 在该反应中, 底物苯酚和频哪醇硼 烷形成保护的苯酚, 其实验和计算研究表明, 在过渡态 中, 底物中的 OBPin 官能团带有部分负电荷, 而联吡啶 配体相对而言具有正电性, 通过过渡态 TS16 实现邻位 碳氢键硼化. 值得注意的是, 要实现苯酚邻位的选择性 嗍化, 苯酚对位通常需要引入大于氟原子的官能团. 在 实验的基础上，作者通过密度泛函理论(DFT)推测出电 性对反应活性与选择性影响, 得到与实验相一致的结 果，进一步证实该过渡态模型的可能性.

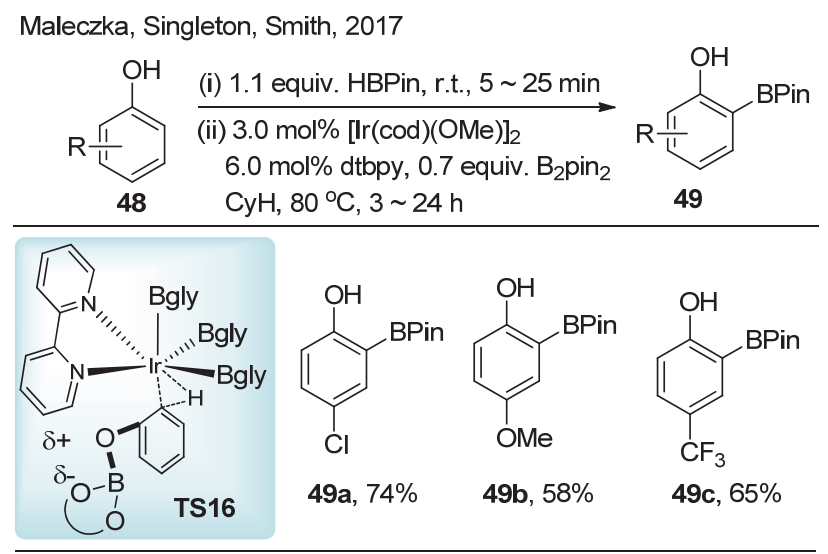

图式 15 静电作用促进的铱催化苯酚邻位碳氢键硼化

Scheme 15 Electrostatic-interaction-enabled Ir-catalyzed ortho-C-H borylation of phenols

\section{3 结论}

近年来，设计和发展基于非共价键作用的配体模板 为选择性碳氢键活化提供了一种全新的模式，通过不同 的非共价键作用，使得催化体系的中心金属、配体模板 及底物分子共同作用, 实现精准可控的选择性活化. 目 前的研究主要集中在氢键作用、离子对作用、路易斯酸 碱对作用和静电作用等. 显而易见的是，该领域尽管已 经有所发展, 并初步显示出其潜在的应用性, 但该领域 仍然存在着一些关键问题岢待解决. (1)目前的报道局限 于铱 $(\mathrm{I})$ 催化的碳氢键嗍化反应, 使用的催化金属为铱 (I), 其他金属尚没有报道，反应类则局限于硼化反应. 这类催化反应的特点是, 整个反应过程为氧化还原中性 体系, 反应体系比较简洁, 无需额外的氧化还原剂和添 加剂. 而众所周知, 绝大多数过渡金属催化的碳氢键活 化对配体、溶剂和添加剂等体系的微调具有极大的依赖 性, 铱(I)催化的非共价键作用的碳氢键硼化得益于联砒 啶类优势骨架配体的应用，而对于其他过渡金属，目前 尚无具有广泛适用性的配体体系得到应用. 另外, 非共 价键作用通常依赖烷烃、甲苯、四氢呋喃和 1,4-二氧六 
环等非极性溶剂, 对反应的适用性也提出了更高的要 求. 其次, 反应体系添加剂, 如酸、碱、无机盐和氧化剂 等, 对非共价键作用体系不可避免地产生一定的影响. 因此，未来在该领域的成功有赖于发展具有促进作用的 普适性配体和温和的反应体系. 最近, $\mathrm{Yu}^{[27]}$ 及 Maiti ${ }^{[28]}$ 课 题组发展了基于钯一氮强配位作用的双功能的模板, 实 现钯催化的杂芳环的选择性碳氢键官能团化, 有望为该 领域的进一步发展提供借鉴. (2)底物类型、选择性、底 物范围和反应类型仍有极大的提升空间 ${ }^{[29]}$. (3)能否发展 手性非共价键的配体模板实现具有前手性化合物的去 对称化和不对称碳氢键活化是该领域的极大挑战 ${ }^{[30]}$. 虽然目前该领域尚处于初级阶段, 随着研究的进一步深 入, 我们相信会有更多的非共价作用模式被开发出来, 为该领域的发展注入蓬勃的生命. 最后, 利用非共价键 作用进行碳氢键的精准官能团化也将为有机合成、药物 开发、超分子化学等领域的发展开启新的篇章.

\section{作者简介}

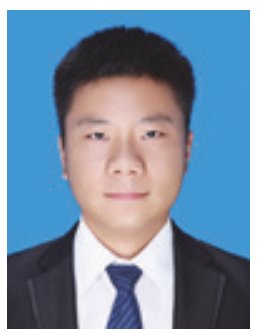

廖港, 博士后, 2018 年毕业于浙江大学, 获理学博士学位, 导师为史炳锋教授. 同年在浙江大学化学系进行博士后研究 工作. 主要研究方向为过渡金属催化不对称碳氢键官能团化.

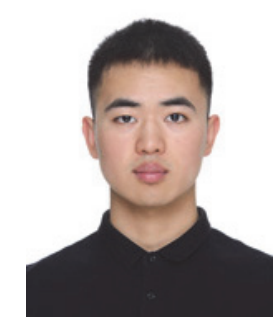

吴勇杰, 2017 年在浙江科技学院获学士学位, 现在在浙江 大学史炳锋教授的指导下攻读博士学位. 主要研究方向为过 渡金属催化不对称碳氢键官能团化.

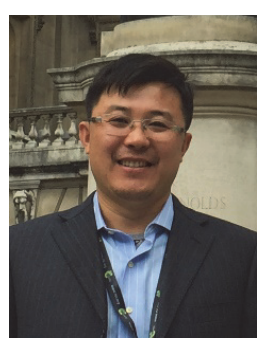

史炳锋, 博士, 教授, 独立课题组组长. 2001 年本科毕业 于南开大学化学系, 2006 年博士毕业于中国科学院上海有机 化学研究所, 导师为俞成研究员. 2006 2007 年在 University of California, San Diego 从事博士后研究, 2007 2010 年加入 The Scripps Research Institute 从事博士后研究, 导师余金权教
授. 2010 年 4 月加入浙江大学化学系, 任独立课题组组长, 博 士生导师。独立工作以来, 以通讯作者发表研究论文九十余 篇, 主要研究领域为过渡金属催化的情性键活化及其在天然 产物全合成中的应用.

\section{References}

[1] For recent reviews on $\mathrm{C}-\mathrm{H}$ activation, see: (a) Chen, $\mathrm{X}$.; Engle, $\mathrm{K}$. M.; Wang, D.-H.; Yu, J.-Q. Angew. Chem., Int. Ed. 2009, 48, 5094. (b) Lyons, T. W.; Sanford, M. S. Chem. Rev. 2010, 110, 1147. (c) Wencel-Delord, J.; Droge, T.; Liu, F.; Glorius, F. Chem. Soc. Rev. 2011, 40, 4740. (d) Pan, F.; Shi, Z. Acta Chim. Sinica 2012, 70, 1679. (潘菲，施章杰，化学学报，2012，70，1679.) (e) Yuan, Y.; Song, S.; Jiao, N. Acta Chim. Sinica 2015, 73, 1231. (袁逸之, 宋颂, 焦宁，化学学报, 2015, 73, 1231.) (f) Zhang, M.; Zhang, Y.; Jie, X.; Zhao, H.; Li, G.; Su, W. Org. Chem. Front. 2014, 1, 843. (g) Xu, J.; Lu, P.; Ye, J.; Liu, G. Acta Chim. Sinica 2015, 73, 1294. (徐佳斌, 陈 品红, 叶金星, 刘国生, 化学学报, 2015, 73, 1294.) (h) Daugulis, O.; Roane, J.; Tran, L. D. Acc. Chem. Res. 2015, 48, 1053. (i) He, G.; Wang, B.; Nack, W. A.; Chen, G. Acc. Chem. Res. 2016, 49, 635. (j) Rao, W.-H.; Shi, B.-F. Org. Chem. Front. 2016, 3, 1028. (k) Yang Y.; Lan, J.; You, J. Chem. Rev. 2017, 117, 8787. (1) He, J.; Wasa, M.; Chan, K. S. L.; Shao, Q.; Yu, J.-Q. Chem. Rev., 2017, 117, 8754. (m) Huang, J.; Gu, Q.; You, S.-L. Chin. J. Org. Chem. 2018, 38, 51. (黄 家翩, 顾庆, 游书力, 有机化学, 2018, 38, 51.) (n) Ren, Q.; Nie, B.; Zhang, Y.; Zhang, J. Chin. J. Org. Chem. 2018, 38, 2465. (任青 云, 聂謎, 张英俊, 张雯, 有机化学, 2018, 38, 2465). (o) Zhao, K.; Yang, L.; Liu, J.; Xia, C. R. Chin. J. Org. Chem. 2018, 38, 2833. (赵 康, 杨砶, 刘建华, 夏春谷, 有机化学, 2018, 38, 2833.) (p) Wang, S.; Yan, F.; Wang, L.; Zhu, L. Chin. J. Org. Chem. 2018, 38, 291. (注珊, 严浲, 汪连生, 朱磊, 有机化学, 2018, 38, 291.) (q) Xu, L.; Xu, H.; Lin, H.; Dai, H. Chin. J. Org. Chem. 2018, 38, 1940. (徐琳 琳, 徐辉, 林海霞, 戴辉雄, 有机化学, 2018, 38, 1940) (r) Gandeepan, P.; Müller, T.; Zell, D.; Cera, G.; Warratz, S.; Ackermann, L. Chem. Rev. 2019, 119, 2192. (s) Zhang, S.; Liao, G.; Shi, B. Chin. J. Org. Chem. 2019, 39, 1522. (张硕, 廖港, 史炳锋, 有机化学, 2019, 39, 1522). (t) Wu, M.; Huang, X.; Zhang, H.; Li, P. Chin. J. Org. Chem. 2019, 39, 3114. (吴梅, 黄新平, 张海兵, 李鹏飞, 有 机化学, 2019, 39, 3114). (u) Zhan, B.; Shi, B.-F. Chin. J. Org. Chem. 2019, 39, 3602. (占贝贝, 史炳锋, 有机化学, 2019, 39, 3602 .

[2] (a) Chen, Z.; Wang, B.; Zhang, J.; Yu, W.; Liu, Z.; Zhang, Y. Org. Chem. Front. 2015, 2, 1107. (b) Sambiagio, C.; Schönbauer, D.; Blieck, R.; Dao-Huy, T.; Pototschnig G.; Schaaf, P.; Wiesinger, T.; Farooq Zia, M.; Wencel-Delord, J.; Besset, T.; Maes, B. U. W.; Schnürch, M. Chem. Soc. Rev. 2018, 47, 6603. (c) Zhang, Q.; Shi, B.-F. Chin. J. Chem. 2019, 37, 647. (d) Rej, S.; Ano, Y.; Chatani, N. Chem. Rev. 2020, 120, 1788.

[3] Zhang, F.-L.; Hong, K.; Li, T.-J.; Park, H.; Yu, J.-Q. Science 2016, $351,252$.

[4] For reviews and representative examples, see: (a) Gong, L.-Z. Acta Chim. Sinica 2018, 76, 817. (龚流柱, 化学学报, Acta Chim. Sinica 2018, 76, 817.) (b) Kim, D.-S.; Park, W.-J.; Jun, C.-H. Chem. Rev. 2017, 117, 8977. (c) Gandeepan, P.; Ackermann, L. Chem 2018, 4 199. (d) John-Campbell, S. S.; Bull, J. A. Org. Biomol. Chem. 2018, 16, 4582. (e) Bhattacharya, T.; Pimparkar, S.; Maiti, D. RSC Adv. 2018, 8, 19456. (f) Qin, Y.; Zhu, L.; Luo, S. Chem. Rev. 2017, 117, 9433. (g) Sun, H.; Guimond, N.; Huang, Y. Org. Biomol. Chem. 2016, 14, 8389. (h) Xu, Y.; Su, T.; Huang, Z.; Dong, G. Angew. Chem., Int. Ed. 2016, 55, 2559. (i) Yao, Q.-J.; Zhang, S.; Zhan, B.-B.; Shi, B.-F. Angew. Chem., Int. Ed. 2017, 56, 6617. (j) Liu, Y.; Ge, H.; Liu, X.-H.; Park, H.; Hu, J.-H.; Hu, Y.; Zhang, Q.-L.; Wang, B.-L.; Sun, B.; Yeung, K.; Zhang, F.-L.; Yu, J.-Q. J. Am. Chem. Soc. 2017, 139, 888. (k) Chen, X. Y.; Ozturk, S.; Sorensen, E. J. Org. Lett. 2017, 19, 1140. (1) Liao, G.; Yao, Q.-J.; Zhang, Z.-Z.; Wu, Y.-J.; Huang, D.-Y.; Shi, B.-F. Angew. Chem., Int. Ed. 2018, 57, 3661. (m) Liao, G.; Li, B.; Chen, H.-M.; Yao, Q.-J.; Xia, Y.-N.; Luo, J.; Shi, B.-F. Angew. Chem. Int. Ed. 2018, 57, 17151. (n) Zhang, S.; Yao, Q.-J.; Liao, G.; Li, X.; Li, H.; Chen, H.-M.; Hong, X.; Shi, B.-F. ACS Catal. 2019, 9, 1956. (o) Song, H.; Li, Y.; Yao, Q.-J.; Jin, L.; Liu, L.; Liu, Y.-H.; Shi, B.-F. Angew. Chem., Int. Ed. 2020, DOI: 10.1002/anie.201915949. (p) Wu, Y.-J.; Yao, Q.-J.; Chen, H.-M.; Liao, G.; Shi, B.-F. Sci. China, Chem. 2020, DOI: 10.1007/s11426020-9694-3. 
[5] Davis, H. J.; Phipps, R. J. Chem. Sci. 2017, 8, 864.

[6] (a) For selected reviews on noncovalent interactions, see: Neel, A. J.; Hilton, M. J.; Sigman, M. S.; Toste, F. D. Nature 2017, 543, 637. (b) Müller-Dethlefs, K.; Hobza, P. Chem Rev. 2000, 100, 143. (c) Breugst, M.; von der Heiden, D.; Schmauck, J. Synthesis 2017, 49, 3224. (d) Hobza P, Müller-Dethlefs K. Non-Covalent Interactions, The Royal Society of Chemistry, Cambridge, 2009. (e) Scheiner, S. Noncovalent Forces, Heidelberg, Springer, 2015; (f) Schreiner, P. R. Chem. Soc. Rev. 2003, 32, 289. (g) Doyle, A. G.; Jacobsen, E. N. Chem Rev. 2007, 107, 5713;

[7] (a) You, C.-C.; Zhang, M.; Liu, Y. Acta Chim. Sinica 2000, 58, 338. (尤长城, 张旻, 刘育, 化学学报, 2000, 58, 338.) (b) Xu, J.; Wang, Z.; Zhang, X. Acta Chim. Sinica 2016, 74, 467. (徐俊, 王治强, 张 希, 化学学报, 2016, 74, 467.) (c) Zhu, J.; Lü, J.-G.; Zhou, Y.-J.; Li, Y.-W.; Chen, J.; Zhen, C.-H. Acta Chim. Sinica 2007, 65, 37. (朱驹, 吕加国, 周有骏, 李耀武, 陈军, 郑灿辉, 化学学报, 2007, 65, 37.) (d) Wheeler, S. E.; Seguin, T. J.; Guan, Y.; Doney, A. C. Acc. Chem. Res. 2016, 49, 1061. (e) Persch, E.; Dumele, O.; Diederich, F. Angew. Chem., Int. Ed. 2015, 54, 3290. (f) Jiang, H.; Li, Q.; Wang, G. Chin. J. Org. Chem. 2018, 38, 1065. (江华, 李巧连, 王光霞, 有 机化学, 2018, 38, 1065.) (g) Jiao, Y.; Zhang, X. Acta Chim. Sinica 2018, 76, 659. (焦阳, 张希, 化学学报, 2018, 76, 659.) (h) Liu, C.-Z.; Wang, H.; Zhang, D.-W.; Zhao, X.; Li, Z.-T. Chin. J. Org. Chem. 2019, 39, 28. (刘传志, 王辉, 张丹维, 赵新, 黎占亭, 有机 化学, 2019, 39, 28.)

[8] Roosen, P. C.; Kallepalli, V. A.; Chattopadhyay, B.; Singleton, D. A.; Maleczka, R. E.; Smith, M. R. J. Am. Chem. Soc. 2012, 134, 11350.

[9] Preshlock, S. M.; Plattner, D. L.; Maligres, P. E.; Krska, S. W.; Maleczka, R. E.; Smith, M. R. Angew. Chem., Int. Ed. 2013, 52, 12915.

[10] Kuninobu, Y.; Ida, H.; Nishi, M.; Kanai, M. Nat. Chem. 2015, 7, 712 .

[11] Wang, J.; Torigoe, T.; Kuninobu, Y. Org. Lett. 2019, 21, 1342.

[12] Lu, X.; Yoshigoe, Y.; Ida, H.; Nishi, M.; Kanai, M.; Kuninobu, Y. ACS Catal. 2019, 9, 1705.

[13] Unnikrishnan, A.; Sunoj, R. B. Chem. Sci. 2019, 10, 3826.

[14] Davis, H. J.; Genov, G. R.; Phipps, R. J. Angew. Chem., Int. Ed. 2017, 56, 13351 .

[15] Davis, H. J.; Mihai, M. T.; Phipps, R. J. J. Am. Chem. Soc. 2016, $138,12759$.

[16] Bai, S.-T.; Bheeter, C. B.; Reek, J. N. H. Angew. Chem., Int. Ed. 2019, 58, 13039.

[17] Mihai, M. T.; Davis, H. J.; Genov, G. R.; Phipps, R. J. ACS Catal. 2018, 8,3764 .
[18] Lee, B.; Mihai, M. T.; Stojalnikova, V.; Phipps, R. J. J. Org. Chem. 2019, 84, 13124.

[19] (a) Mihai, M.; Williams, B. D.; Phipps, R. J. J. Am. Chem. Soc. 2019, 141, 15477. (b) Montero Bastidas, J. R.; Oleskey, T. J.; Miller S. L.; Smith, M. R.; Maleczka, R. E. J. Am. Chem. Soc. 2019, 141, 15483 .

[20] Bisht, R.; Chattopadhyay, B. J. Am. Chem. Soc. 2016, 138, 84

[21] Li, H. L.; Kuninobu, Y.; Kanai, M. Angew. Chem., Int. Ed. 2017, 56, 1495.

[22] Yang, L.; Semba, K.; Nakao, Y. Angew. Chem., Int. Ed. 2017, 56, 4853 .

[23] Yang, L.; Uemura, N.; Nakao, Y. J. Am. Chem. Soc. 2019, 141, 7972.

[24] Hoque, M. E.; Bisht, R.; Haldar, C.; Chattopadhyay, B. J. Am. Chem. Soc. 2017, 139, 7745.

[25] Bisht, R.; Hoque, M. E.; Chattopadhyay, B. Angew. Chem., Int. Ed. 2018, 57, 15762 .

[26] Chattopadhyay, B.; Dannatt, J. E.; Andujar-De Sanctis, I. L.; Gore, K. A.; Maleczka, R. E.; Singleton, D. A.; Smith, M. R. J. Am. Chem. Soc. 2017, 139, 7864.

[27] Zhang, Z.; Tanaka, K.; Yu, J.-Q. Nature 2017, 543, 538.

[28] (a) Achar, T. K.; Ramakrishna, K.; Porey, S.; Pal, T.; Dolui, P.; Biswas, J. P.; Maiti, D. Chem.-Eur. J. 2018, 24, 17906. (b) Ramakrishna, K.; Biswas, J. P.; Jana, S.; Achar, T. K.; Porey, S.; Maiti, D. Angew. Chem., Int. Ed. 2019, 58, 13808.

[29] Haldar, C.; Hoque, M. E.; Bisht, R.; Chattopadhyay, B. Tetrahedron Lett. 2018, 59, 1269.

[30] (a) Giri, R.; Shi, B.-F.; Engle, K. M.; Maugel, N.; Yu, J.-Q. Chem. Soc. Rev. 2009, 38, 3242. (b) Wencel-Delord, J.; Colobert, F. Chem.Eur. J. 2013, 19, 14010. (c) Zheng, C.; You, S.-L. RSC Adv. 2014, 4 6173. (d) Gao, D.-W.; Gu, Q.; Zheng, C.; You, S.-L. Acc. Chem. Res. 2017, 50, 351. (e) Newton, C. G.; Wang, S.-G.; Oliveira, C. C.; Cramer, N. Chem. Rev. 2017, 117, 8908. (f) Yan, S.-Y.; Han, Y.-Q.; Yao, Q.-J.; Nie, X.-L.; Liu, L.; Shi, B.-F. Angew. Chem., Int. Ed. 2018, 57, 9093. (g) Saint-Denis, T. G.; Zhu, R.-Y.; Chen, G.; Wu, Q.-F.; Yu, J.-Q. Science 2018, 359, 759. (h) Liao, G.; Zhou, T.; Yao, Q.-J.; Shi, B.-F. Chem. Commun. 2019, 55, 8514. (i) Han, Y.-Q.; Ding, Y.; Zhou, T.; Yan, S.-Y.; Song, H.; Shi, B.-F. J. Am. Chem. Soc. 2019, 141, 4558. (j) Luo, J.; Zhang, T.; Wang, L.; Liao, G.; Yao, Q.-J.; Wu, Y.-J.; Zhan, B.-B.; Lan, Y.; Lin, X.-F.; Shi, B.-F. Angew. Chem., Int. Ed. 2019, 58, 6708. (k) Zhan, B.-B.; Wang, L.; Luo, J.; Shi, B.-F. Angew. Chem., Int. Ed. 2020, 59, 3568. (1) Zhou, T.; Jiang, M.-X.; Yang, X.; Yue, Q.; Han, Y.-Q.; Ding, Y.; Shi, B.-F. Chin. J. Chem. 2020, 38, 242. 